\title{
Divisions I \& III WG: on Near Earth Objects
}

\author{
CHAIRPERSON: David Morrison \\ VICE-CHAIRPERSON: Andrea Milani \\ ORGANIZING COMMITTEE: Richard Binzel, Ted Bowell, \\ Andrea Carusi, Clark Chapman, Alan Harris, Syuzo Isobe, \\ Brian Marsden, Karri Muinonen, Steve Ostro, Victor Shor, \\ Duncan Steel, Gonzalo Tancredi, Jana Ticha, Giovanni Valsecchi, \\ and Don Yeomans
}

\section{Introduction}

The WGNEO, a Working Group of Divisions I and III, was formed in the early 1990s to coordinate study of Earth-approaching asteroids and comets (NEOs) and provide timely advice to the General Secretary and officers of the IAU on discovery of any objects that threaten collision with the Earth. Since then, the WGNEO has steadily grown, reflecting increasing international interest and concern over impacts, especially from asteroids (which dominate over comets in their risk to Earth). In this triennium, the WGNEO had a membership of 49 (including the Organizing Committee of 17 members), plus 10 consultants. The Chair is David Morrison (USA), Vice-Chair Andrea Milani (Italy), Secretary Richard Binzel (USA), and Past-Chair Andrea Carusi (Italy).

\section{Report}

Much of the recent work of the WGNEO has focused on the assessment of risk and its communication to the media and the public. As new NEOs are discovered (now several per day) and their orbits are calculated, astronomers are becoming more experienced in the prediction of their future paths. For time after discovery (usually no more than a few days), the uncertainties in the predicted orbit may indicate one or more low-probability impacts. While the results of the surveys and orbit calculations are posted on the Internet, the communication of these results remains a challenge. The WGNEO has adopted the 10-point Torino Scale as its primary means to inform the public of the danger posed by any specific asteroid and impact opportunity. Increasing use of the Torino Scale, together with more sophisticated journalists, has reduced the number of media alarms during this triennium in spite of a steadily increasing discovery rate.

Highlights of this triennium follow. Expansion of search capability by the University of Arizona team led by Steven Larson has added to the already impressive survey work of the LINEAR team (Grant Stokes) to provide 4 state-of-the-art 1-m Spaceguard Survey telescopes, which together generate 80 .

Asteroid Apophis (originally 2004 MN4) was the subject of extensive study and wide publicity due to its extremely close pass by Earth predicted for 2029 April 13; for a few days in December 2004 it reached Torino Scale 4, two steps higher than any previous NEO. Subsequent Apophis studies have focused on a continuing small risk of impact in April 2036, and Apophis has stimulated much theoretical work on keyholes, which are points in space through which a NEO may pass that lead to impact on subsequent close 
approaches. In 2006, asteroid 2004 VD17 reached Torino Scale 2. As NEO discovery rates increase, we can expect many future cases where there is a significant chance of collision with Earth. At the end of the current triennium, approximately 840 NEAs larger than $1 \mathrm{~km}$ diameter had been discovered by the Spaceguard Survey, representing almost 80 percent of this population. An additional 3000 sub-km NEAs have been discovered.

The issue of defending against asteroids has been discussed by the United Nations, and draft treaty language is being considered. The IAU has played an important role in bringing the asteroid impact issue before the Organization for Economic Co-operation and Development Global Science Forum, and the International Council of Scientific Unions. In early 2006 the United States Congress revised the NASA charter to include study of potentially hazardous asteroids, and a plan for a NASA NEO program is to be submitted to Congress in December 2006.

Following on the successful orbit and landing on the surface of Eros by the NASA NEAR-Shoemaker spacecraft, the era of space exploration has begun. In 2006, the Japanese Hyabusa mission studied the sub-km NEO Itokawa at extreme close range, while the NASA Deep Impact mission demonstrated the first ballistic impact with a comet. The ESA Don Quijote mission, currently under study, will test technologies for deflection on a sub-km asteroid.

The WGNEO is discontinued. Consideration will be given in the future to the role of existing Commissions in Division III to support international coordination of NEO searches and studies. To provide timely information to the IAU officers on NEO issues, a new 5-person NEO Committee is proposed, reporting to the General Secretary and President of the Union. 